\title{
A multi-instance multi-sample palmprint identification system
}

\author{
Thulfiqar H. Mandeel, Muhammad Imran Ahmad, Said Amirul Anwar \\ School of Computer and Communication Engineering, Universiti Malaysia Perlis, Malaysia
}

\begin{tabular}{l} 
Article Info \\
\hline Article history: \\
Received Oct 1, 2018 \\
Revised Nov 27, 2018 \\
Accepted Dec 12, 2018 \\
\hline
\end{tabular}

\section{Keywords:}

Biometrics recognition

Fusion

Palmprint

\begin{abstract}
The multibiometric recognition system considered more reliable than the unimodal biometric recognition system due to the addition of an extra information that increases the discrimination between the classes. In this paper, a multi-sample multi-instance biometric recognition system is proposed. The aim of the proposed system is to increase the robustness of the identification. The proposed system also addresses the overfitting to the train samples problem of a feature extraction algorithm, named 2-Dimensional Linear Discriminant analysis (2D-LDA). The samples in the proposed method are bootstrapped and the 2D-LDA performed on each group during the offline phase. While in the online phase, the tested class will be transformed into subspaces using different eigenvectors that obtained from different samplings, and the results matched with templates in the corresponding subspace. To evaluate the proposed method, two palmprint databases are used which are IIT Delhi Touchless Palmprint Database and PolyU palmprint database, and different rank-level fusion algorithms are investigated. The results of the proposed method show improvement in the identification rate.
\end{abstract}

Copyright (c) 2019 Institute of Advanced Engineering and Science. All rights reserved.

\footnotetext{
Corresponding Author:

Muhammad Imran Ahmad,

Universiti Malaysia Perlis,

Kampus Pauh Putra, 02600 Arau, Perlis, Malaysia.

Email: m.imran@unimap.edu.my
}

\section{INTRODUCTION}

Human's physical and behavioral characteristics differ from an individual to another which give us the ability to distinguish a person from another. Nevertheless, this natural and accurate human skill is affected by memory limits which mean its inapplicable to memorize a large database of distinctive biometric features from palmprint or iris for example. Automatic biometric recognition systems are devised to overcome such problem [1], [2]. The most common human characteristics that can be used in biometric recognition systems are physical characteristics such as the face, Iris, fingerprint, palmprint and hand geometry, or behavioral characteristics such as speech, gait, keystroke, and signature. Furthermore, these characteristics vary in different aspects: accuracy, universality, distinctiveness, permanence, collectability, and acceptability. Among these characteristics, palmprint show high distinctiveness and fair acceptability [1].

However, the implementation of single biometric characteristics in a unimodal system (e.g. single sample, single fingerprint) has a limitation in the recognition accuracy due to the limited amount of the extracted information which affect the system performance [3], [4]. Multibiometric recognition systems have been emerged to solve this issue in the unimodal systems. Multimodal systems can be formed using multiple sensors, multiple samples, multiple instances, or multiple algorithms [3]. The information from these sources can be fused at different stages of the biometric recognition system. The fusion can be done before the matching: at sensor-level [5], [6] or at feature-level [7]-[9], or after the matching: at score-level [10]-[12], ranklevel [13]-[15] or at decision-level [16]-[19]. Nevertheless, the use of multiple sensors system raises the cost of the biometric recognition system [4]. The multi-algorithms approach is proposed to obtain extra information from the same biometric data [20], [21] which eliminate the need for the extra acquisition equipment. However, this approach consumes substantial time because different feature extraction algorithms need to be applied first 
and different type of classifiers may be needed depending on the nature of the extracted features. To fix this issue, Yan et al. [4] proposed extracting the same feature (i.e. SIFT feature) from multiple samples of palm vein images and fuses them at the feature-level during the offline phase for robust identification. Leng et. al. [22] merged 2D-DCT features from left and right palm at feature-level. While at the score-level, Cheng et al. [23] fused the score of matching from the repeated scan of fingerprints (i.e., multi-sample) during the online phase. Kumar and Shekhar [14] argued that the rank-level fusion is preferable over other fusion levels for multibiometric identification due to firstly, elimination of feature or score normalization, secondly, provide device and algorithms independency, thirdly, the actual identification may fall in second or third rank especially in large database due to limitation in the feature extraction algorithm or/and classifier efficiency.

There are two common algorithms for feature extraction, named Linear Discriminant Analysis (LDA) and Principal Component Analysis (PCA). These algorithms require the image to be in 1-dimensional form before being feed into the PCA or the LDA, which is memory expensive. These algorithms also prone to the small sample size (SSS) problem, which means many samples required to extract reliable representation. Yang et al. [24] and Li \& Yuan [25] proposed using the original images without converting them into one vector, which is more efficient. Another approach was proposed to overcome the SSS problem and reduce the overfitting to the training set by Nguyen et al. [26] and Wang \& Tang [27]. These algorithms propose selecting the significant eigenvectors and randomly select other less important eigenvectors to make several weak classifiers and combine them to make better classifier. The eigenvectors that correspond to big eigenvalues are selected because they are better in discriminating the classes, in the LDA algorithm, or better in representing the data, in the PCA algorithm. However, the random selection of eigenvectors may lead to the inclusion of a noise or insignificant data which result in degraded performance [28].

In the proposed method the eigenvectors will be selected according to their significance instead of the random selection. Moreover, to avoid overfitting, the samples are bootstrapped, and the 2D-LDA applied to the different formations of training samples and different eigenvectors extracted from each formation. Later, these eigenvectors will be used to transform another sample that dedicated for testing into different subspaces to generate templates and save them in the system database during offline phase. While in the online phase, these different eigenvectors will also be used to transform the tested palmprint image into different subspaces and match the results with the classes in the corresponding subspaces. The results from these matches will be fused to find the final palmprint identity at rank-level. The general outline of the proposed method presented in Figure 1.

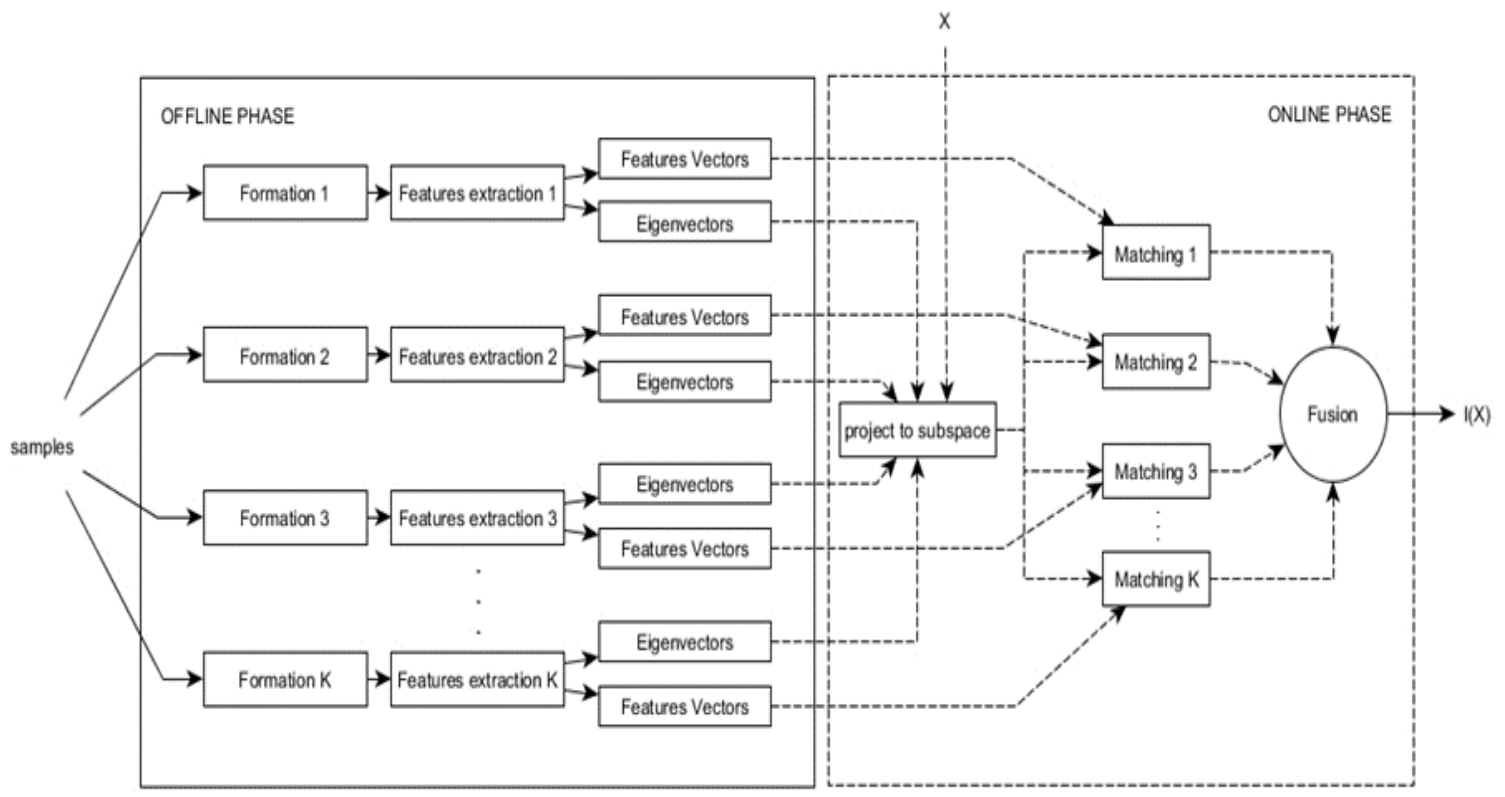

Figure 1. The proposed method

The rest of the paper is organized as follow: sections 2 represent the proposed method; section 3 shows the result and the experiment setup while section 4 represents the conclusion. It's should be mentioned that the Maximum Rank Method and the majority voting scheme isn't implemented due to the generation of ties which randomly broken to produce the tested class identity [13]. 


\section{THE PROPOSED METHOD}

The first step in the proposed method is dividing the classes' samples $G_{1}, \ldots, G_{k}$ into two groups: the train group $G_{L}$ and the test group $G_{T}$, the train group has $c$ classes and $n$ samples, $G_{L_{c, n}}$, while the test group has one sample $G_{T_{C, 1}}$. During the offline phase, the 2D-LDA applied on $G_{L}$ to extract the eigenvectors and these eigenvectors used to reduce the dimensionality of $G_{T}$ and generate the templates. The selection of the samples to be included in $G_{L}$ and $G_{T}$ will vary to generate $k$ different formations, $G_{L_{c, n}}^{k}$ and $G_{T_{c, 1}}^{k}$, as shown in Figure 2.

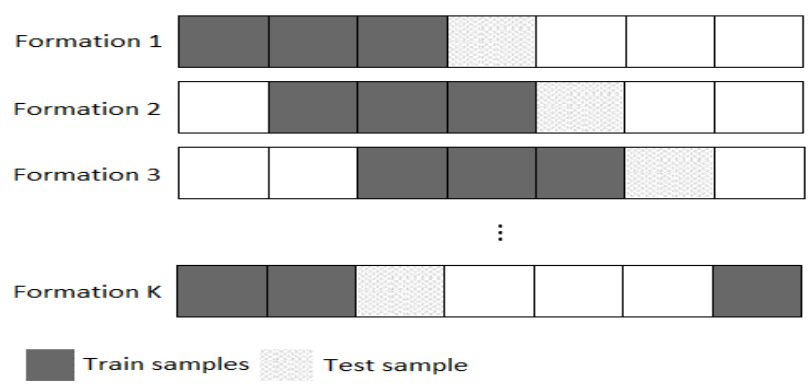

Figure 2. The Different Formations of $G_{L}$ and $G_{T}, k$ here is 7

The proposed method is represented mathematically as follow:

$$
\boldsymbol{S}_{\boldsymbol{w}}^{\boldsymbol{k}}=\sum_{i=1}^{c} \sum_{G_{L_{c, n}} \in D_{i}}^{n}\left(\boldsymbol{G}_{L_{c, n}}^{k}-\boldsymbol{M}_{i}\right)\left(\boldsymbol{G}_{L_{c, n}}^{k}-\boldsymbol{M}_{i}\right)^{T}
$$

where $\boldsymbol{S}_{W}$ is scatter matrix within classes, $\boldsymbol{M}_{i}$ is the mean for each class, $\boldsymbol{G}_{L_{c, n}}^{k}$ is 2-dimensional palmprint image. $n$ is the number of the samples for each class in $G_{L}$.

$$
\begin{aligned}
\boldsymbol{M}_{i} & =\frac{1}{n_{i}} \sum_{G_{L_{c, n}} \epsilon D_{i}}^{n} \boldsymbol{G}_{L_{c, n}}^{k} \\
\boldsymbol{S}_{\boldsymbol{B}}^{\boldsymbol{k}} & =\sum_{i=1}^{c} N_{i}\left(\boldsymbol{M}_{i}-\boldsymbol{M}\right)\left(\boldsymbol{M}_{i}-\boldsymbol{M}\right)^{T}
\end{aligned}
$$

where $\boldsymbol{S}_{B}$ is the scatter matrix between classes $m$ is the overall mean, and $m_{i}$ and $N_{i}$ are the mean and size of the respective class $i$. The final scatter matrix is evaluated as in the equation:

$$
A^{k}=\boldsymbol{S}_{w}^{k-1} \boldsymbol{S}_{\boldsymbol{B}}^{k}
$$

Finally, the eigenvector and eigenvalue are calculated as

$$
A^{k} v^{k}=\omega^{k} v^{k}
$$

Where $v$ is $1 \times b$ eigenvector and $\omega$ is the eigenvalue. To create the new subspace, $h$ eigenvectors with corresponding high eigenvalues are selected $v_{1}, \ldots, v_{h}$. and each vector is multiplied with original images from the test group to form a feature vector.

$$
Y_{d_{c}}^{k}=v_{d}^{k} * G_{T_{C, 1}}^{k}, d=1, \ldots, h
$$

The templates are constructed from the acquired feature vectors as:

$$
M L_{c}^{k}=\left[Y_{1_{c}}^{k}, \ldots, Y_{d_{c}}^{k}\right]
$$

In the online phase, the tested class transformed into subspace using the $v_{d}^{k}$ to generate feature matrices $M T^{k}$. To determine the tested class identity, the Euclidean distance is calculated between the $M T^{k}$ and $M L_{c}^{k}$. 


$$
D^{k}\left(M T^{k}, M L_{c}^{k}\right)=\sqrt{\sum_{i=1}^{b}\left(M T_{i}^{k}-M L_{c_{i}}^{k}\right)^{2}}
$$

Lastly, different fusion schemes can be implemented using $D^{k}$ to obtain accurate class identity.

\section{RESULTS AND EXPERIMENT SETUP}

To evaluate the proposed method, the experiments conducted on two palmprint databases which are the IIT Delhi Touchless Palmprint Database and PolyU palmprint database. Different number of samples of palmprint images included in $G_{L}$ as available in the corresponding database. The votes are obtained by order the values of $D \forall k$ in ascending fashion. IIT Delhi Touchless Palmprint Database includes images from left and right hands that gathered from 460 palms in a peg free form. The region of interest (ROI) cropped automatically and included in the database which has the size of $150 * 150$ pixels [29]. For each palm, four samples out of five used to form four formations. The noise that presents in IIT Delhi palmprint database in the form of distortion and translation considered as a factor that degrades the recognition rate if a single sample single-instance is used. PolyU palmprint database includes 7,752 palmprint images belongs to 386 palms which gathered using charge-coupled device (CCD) camera-based device with the help of pegs to fix palm position which minimizes the spatial distortion between the images that belongs to the same palm. The images have been collected through out two sessions. To mimic the real-world-scenario, these two sessions separated by around two months. In these two sessions, most of the subjects provide around ten images for left and right palms separately [30]. To keep the uniformity between the classes, the palmprints that have at less seven samples included in our experiment which can be summed to 378 different palms. These palms are divided randomly into two categories to represent the left and right palms by which each category has 189 palms. The ROIs for this database obtained using the method proposed in [31].

The fusion at score-level is not applicable for left and right palmprints fusion because palmprints for the right and left palms that belong to the same subject are not identical. Hence it is inapplicable to fuse the matching score from corresponding palms without former knowledge of the correlation between them. Xu ET. al. [32] showed a correlation between the principal lines of the left and right palms that belongs to the same person. Nevertheless, their experiment showed the best results when assigning a small weight for the fused scores from cross palms $(w \leq 0.1)$.

The algorithms that implemented in this paper for rank fusion are Borda Count and Bucklin Majority Voting, both with different rank levels. In the Borda Count Method, scores are assigned to every identity that produced by each classifier. The identity at the first rank receives highest points, while identities at lower ranks receive lower points according to their level. Later, these points are summed and the identity with the highest points will be considered as genuine identity [13]. In Bucklin Majority Voting, the first rank will be used to extract the identity votes and the class with the majority of votes is declared as the genuine identity. If there is no majority produced, the votes from other ranks will be included in a steps fashion depend on the mentioned condition. Table 1 and Table 2 show the identification rate, the average identification rate is obtained from averaging the results from the unimodal model at different samples formations, these results obtained using 2D-LDA [25]. The number of the used ranks is between parentheses, while the number of ranks has a positive impact on the identification rate (for both palms) of PolyU database it has the opposite on the IIT Delhi database. The reason for such effect is that IIT Delhi classes have a clear intraclass variation in form of rotation, perspective, or distance from the camera, which reduce the discrimination power and result in scattered genuine identities along the different ranks. Also, the inclusion of more ranks can degrade the performance and increase the confusion in the final decision.

Table 1. Recognition Rate (RR) for IIT Delhi database

\begin{tabular}{|c|c|c|c|c|c|c|c|c|}
\hline $\begin{array}{l}\text { Number } \\
\text { of train } \\
\text { images }\end{array}$ & $\begin{array}{c}\text { Average of } \\
\text { left palm } \\
\text { RR }(\%)\end{array}$ & $\begin{array}{l}\text { Average of } \\
\text { right palm } \\
\text { RR }(\%)\end{array}$ & $\begin{array}{c}\text { Borda } \\
\text { Count/left } \\
\text { palms RR } \\
(\%)\end{array}$ & $\begin{array}{c}\text { Borda } \\
\text { Count/right } \\
\text { palms RR } \\
(\%)\end{array}$ & $\begin{array}{c}\text { Borda } \\
\text { Count/both } \\
\text { palms RR }(\%)\end{array}$ & $\begin{array}{c}\text { Bucklin } \\
\text { Majority } \\
\text { Voting/ left } \\
\text { palms RR } \\
(\%)\end{array}$ & $\begin{array}{l}\text { Bucklin } \\
\text { Majority } \\
\text { Voting/ } \\
\text { tight palms } \\
\text { RR }(\%)\end{array}$ & $\begin{array}{c}\text { Bucklin } \\
\text { Majority } \\
\text { Voting/ both } \\
\text { palms RR } \\
(\%)\end{array}$ \\
\hline 2 & 55.9 & 61.7 & & & & & & \\
\hline $2(1)$ & & & 65.6 & 68.6 & 86 & & & \\
\hline $2(3)$ & & & 54.7 & 58.6 & 76.5 & & & \\
\hline $3(1)$ & & & 66.5 & 67.3 & 84.7 & & & \\
\hline $3(3)$ & & & 56.9 & 62.1 & 79.5 & & & \\
\hline $3(6)$ & & & 55.2 & 60.8 & 77.3 & 61.3 & 65.21 & 82.1 \\
\hline
\end{tabular}


Table 2. Recognition Rate (RR) for PolyU palmprint database

\begin{tabular}{|c|c|c|c|c|c|c|c|c|}
\hline $\begin{array}{l}\text { Number } \\
\text { of train } \\
\text { images }\end{array}$ & $\begin{array}{l}\text { Average } \\
\text { of left } \\
\text { palm RR } \\
(\%)\end{array}$ & $\begin{array}{c}\text { Average } \\
\text { of right } \\
\text { palm RR } \\
(\%)\end{array}$ & $\begin{array}{c}\text { Borda } \\
\text { Count/left } \\
\text { palms RR } \\
(\%)\end{array}$ & $\begin{array}{c}\text { Borda } \\
\text { Count/right } \\
\text { palms RR } \\
(\%)\end{array}$ & $\begin{array}{c}\text { Borda } \\
\text { Count/both } \\
\text { palms RR } \\
(\%)\end{array}$ & $\begin{array}{c}\text { Bucklin } \\
\text { Majority } \\
\text { Voting/ left } \\
\text { palms RR } \\
(\%)\end{array}$ & $\begin{array}{l}\text { Bucklin } \\
\text { Majority } \\
\text { Voting/ } \\
\text { right palms } \\
\text { RR }(\%)\end{array}$ & $\begin{array}{c}\text { Bucklin } \\
\text { Majority } \\
\text { Voting/ } \\
\text { both palms } \\
\text { RR }(\%)\end{array}$ \\
\hline 2 & 95.2 & 95.4 & & & & & & \\
\hline $2(1)$ & & & 97.8 & 97.3 & 98.4 & & & \\
\hline $2(3)$ & & & 97.8 & 97.3 & 100 & & & \\
\hline $2(6)$ & & & 96.8 & 97.3 & 100 & 97.3 & 97.8 & 98.9 \\
\hline 3 & 95.1 & 95.2 & & & & & & \\
\hline $3(1)$ & & & 97.8 & 97.3 & 98.4 & & & \\
\hline $3(3)$ & & & 97.3 & 96.2 & 100 & & & \\
\hline $3(6)$ & & & 96.8 & 96.2 & 100 & 98.4 & 97.3 & 98.9 \\
\hline 4 & 95.3 & 95.1 & & & & & & \\
\hline $4(1)$ & & & 96.2 & 97.8 & 98.9 & & & \\
\hline $4(3)$ & & & 95.2 & 95.2 & 100 & & & \\
\hline $4(6)$ & & & 94.1 & 94.1 & 100 & 96.8 & 96.8 & 99.4 \\
\hline 5 & 95.2 & 94.8 & & & & & & \\
\hline $5(1)$ & & & 95.2 & 96.8 & 98.9 & & & \\
\hline $5(3)$ & & & 93.1 & 94.7 & 100 & & & \\
\hline $5(6)$ & & & 93.1 & 95.7 & 100 & 94.7 & 95.2 & 99.4 \\
\hline
\end{tabular}

\section{CONCLUSION}

In this paper a new method proposed for a multi-sample multi-instance palmprint recognition system. In the proposed method, the palmprint images samples that used for training were selected differently prior to the training process. The proposed method fixed two problems, the first one is the overfitting to the train data. While the second problem is the limited discrimination information in the unimodal biometric recognition systems. The results indicate that the rising of the discrimination information lead to an improvement in the recognition accuracy and restraining the noise. These solutions achieved without implementation for extra algorithms or sensors.

\section{ACKNOWLEDGMENTS}

This research is supported partially by graduate assistant (GA) fund from Universiti Malaysia Perlis (UniMAP) with reference number: UniMAP/PPPI/1-18/JId. 2. This research is supported by Ministry of Education Malaysia financial under the Fundamental Research Grant Scheme (FRGS) Grant No: 9003-00583

\section{REFERENCES}

[1] A. K. Jain, et al., "An Introduction to Biometric Recognition," IEEE Trans. Circuits Syst. Video Technol., vol. 14, pp. 4-20, 2004.

[2] J. Wayman, et al., "An Introduction to Biometric Authentication Systems," Biometric Syst., pp. 1-20, 2005.

[3] L. M. Dinca and G. P. Hancke, "The Fall of One, the Rise of Many: A Survey on Multi-Biometric Fusion Methods," IEEE Access, vol. 5, pp. 6247-6289, 2017.

[4] X. Yan, et al., "Palm vein recognition based on multi-sampling and feature-level fusion," Neurocomputing, vol. 151, pp. 798-807, 2015.

[5] S. Shah, et al., "Fingerprint Mosaicing using Thin Plate Splines," Proc. Biometrics Consort. Conf. BCC 2005, pp. 12, 2005.

[6] G. P. Kusuma and C. S. Chua, "PCA-based image recombination for multimodal 2D + 3D face recognition," Image Vis. Comput., vol. 29, pp. 306-316, 2011.

[7] G. U. Bokade and A. M. Sapkal, "Feature Level Fusion of Palm and Face for Secure Recognition," Int. J. Comput. Electr. Eng., vol. 4, pp. 157-160, 2012.

[8] M. S. Almohammad, et al., "Human Identification System Based on Feature Level Fusion using Face and Gait Biometrics," Eng. Technol., pp. 2-6, 2012.

[9] U. Gawande, et al., "A Novel Algorithm for Feature Level Fusion Using SVM Classifier for Multibiometrics-Based Person Identification," Appl. Comput. Intell. Soft Comput., vol. 2013, pp. 1-11, 2013.

[10] M. Hanmandlu, et al., "Score level fusion of multimodal biometrics using triangular norms," Pattern Recognit. Lett., vol. 32, pp. 1843-1850, 2011.

[11] H. F. Liau and D. Isa, "Feature selection for support vector machine-based face-iris multimodal biometric system," Expert Syst. Appl., vol. 38, pp. 11105-11111, 2011.

[12] S. Aoyama, et al., "Similarity measure using local phase features and its application to biometric recognition," IEEE Comput. Soc. Conf. Comput. Vis. Pattern Recognit. Work., pp. 180-187, 2013.

[13] A. Kumar, "Rank Level-Fusion," in Encyclopedia of Biometrics, S. Z. Li, A. Jain, Eds. Boston, MA: Springer, 2009, pp. 607-611. 
[14] A. Kumar and S. Shekhar, "Personal identification using multibiometrics rank-level fusion," IEEE Trans. Syst. Man Cybern. Part C Appl. Rev., vol. 41, pp. 743-752, 2011.

[15] A. Abaza and A. Ross, "Quality based rank-level fusion in multibiometric systems," IEEE 3rd Int. Conf. Biometrics Theory, Appl. Syst. BTAS 2009, pp. 1-6, 2009.

[16] X. Ren, et al., "Multi-fingerprint information fusion for personal identification based on improved dempster-shafer evidence theory," Proc. Int. Conf. Electron. Comput. Technol., pp. 281-285, 2009.

[17] A. Kumar, et al., "Decision level biometric fusion using ant colony optimization," Image Process., pp. 3105-3108, 2010.

[18] P. P. Paul, et al., "Decision Fusion for Multimodal Biometrics Using Social Network Analysis," IEEE Trans. Syst. Man Cybern. Syst., vol. 44, pp. 1522-1533, 2014.

[19] M. Abdolahi, et al., "Multimodal Biometric system Fusion Using Fingerprint and Iris with Fuzzy Logic," Int. J. Soft Comput. Eng., vol. 2, pp. 504-510, 2013.

[20] J. Yang and X. Zhang, "Feature-level fusion of global and local features for finger-vein recognition," Int. Conf. Signal Process. Proceedings, ICSP, pp. 1702-1715, 2010.

[21] X. Qian, et al., "Finger-vein recognition based on the score level moment invariants fusion," Proc. - 2009 Int. Conf. Comput. Intell. Softw. Eng. CiSE 2009, pp. 3-6, 2009.

[22] L. Leng, et al., "Dual-source discrimination power analysis for multi-instance contactless palmprint recognition," Multimed. Tools Appl., vol. 76, pp. 333-354, 2017.

[23] X. Cheng, et al., "Multiple-sample fusion of matching scores in biometric systems," IEEE Comput. Soc. Conf. Comput. Vis. Pattern Recognit. Work., 2011.

[24] J. Yang, et al., "Two-Dimensional PCA: A New Approach to Appearance-Based Face Representation and Recognition," IEEE Trans. Pattern Anal. Mach. Intell., vol. 26, pp. 131-137, 2004.

[25] M. Li and B. Yuan, "2D-LDA: A statistical linear discriminant analysis for image matrix," Pattern Recognit. Lett., vol. 26, pp. 527-532, 2005.

[26] N. Nguyen, et al., "Random Subspace Two-Dimensional PCA for Face Recognition," Compute, pp. 655-664, 2007.

[27] X. Wang and X. Tang, "Random sampling LDA for face recognition," in Proceedings of the 2004 IEEE Computer Society Conference on Computer Vision and Pattern Recognition, 2004. CVPR 2004., 2004, vol. 2, pp. 259-265.

[28] A. M. Martinez and A. C. Kak, "PCA versus LDA," IEEE Trans. Pattern Anal. Mach. Intell., vol. 23, pp. 228-233, 2001.

[29] A. Kumar and D. Zhang, "Personal Recognition Using Hand Shape and Texture," IEEE Trans. Image Process., vol. 15, pp. 2454-2461, 2006.

[30] D. Zhang, et al., "Online palmprint identification," IEEE Trans. Pattern Anal. Mach. Intell., vol. 25, pp. 1041-1050, 2003.

[31] T. H. Mandeel, et al., "Palmprint Region of Interest Cropping Based on Moore-Neighbor Tracing Algorithm," Sens. Imaging, vol. 19, pp. 1-14, 2018.

[32] Y. Xu, et al., "Combining left and right palmprint images for more accurate personal identification," IEEE Trans. Image Process., vol. 24, pp. 549-559, 2015.

\section{BIOGRAPHIES OF AUTHORS}

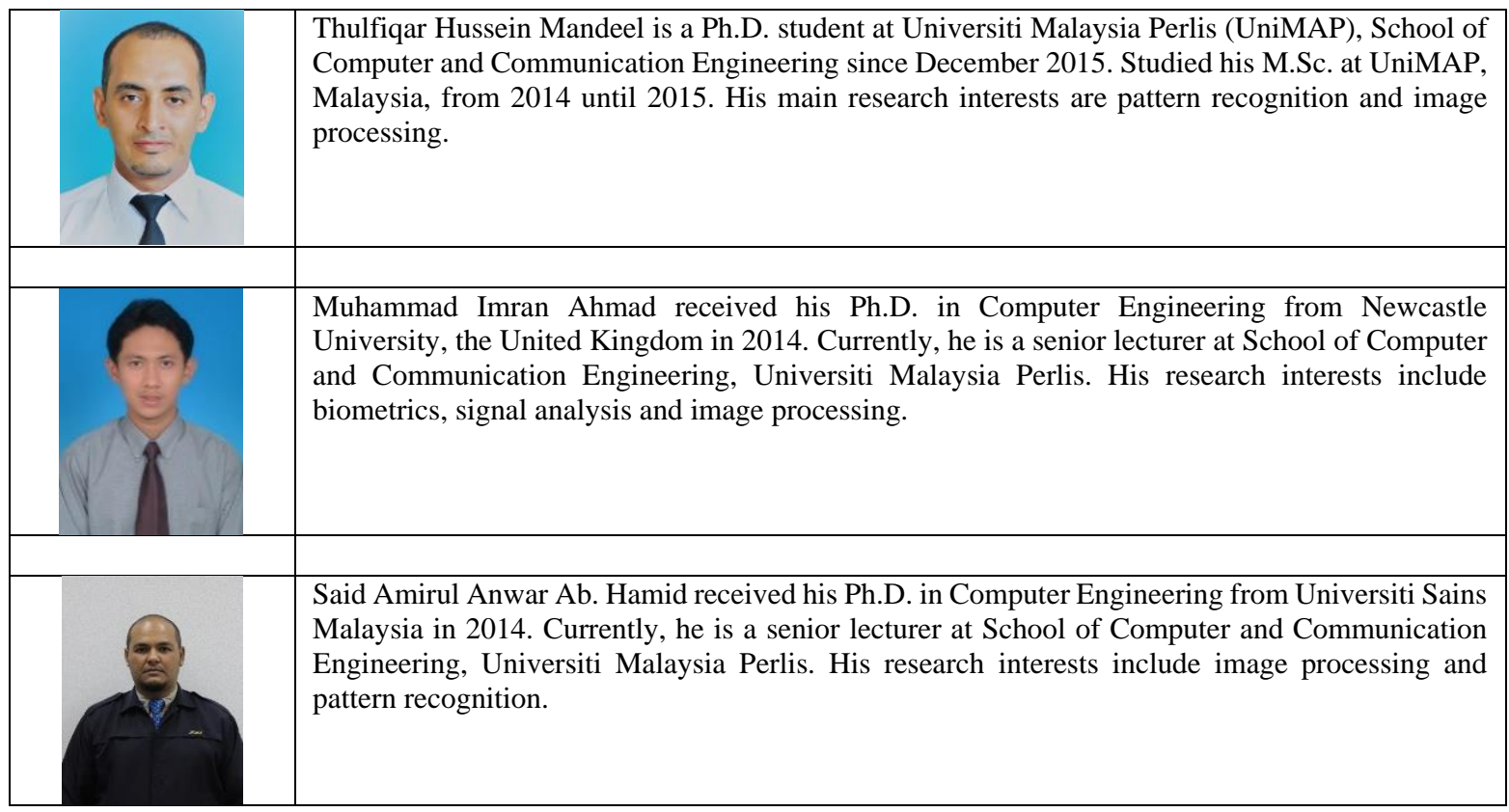

